\title{
Hyphenation of aqueous two-phase and microwave extraction of solasonine and solamargine from leaves of Solanum mauritianum characterized by UHPLC-qTOF-MS
}

\author{
Tebogo Mphatlalala Mokgehle ${ }^{1}$ (D) $\cdot$ Ntakadzeni Edwin Madala $^{2} \cdot$ Wilson Mugera Gitari $^{3} \cdot$ Nikita Tawanda Tavengwa $^{1}$
}

Received: 26 July 2021 / Revised: 9 November 2021 / Accepted: 24 November 2021

(c) The Author(s) 2021

\begin{abstract}
The biomass Solanum mauritianum (S. mauritianum) is an invasive weed specie; however, it is a source of medicinally important metabolites, as reported in literature, such as solasonine and solamargine. The study was directed at the optimization of microwave and aqueous two-phase-based extraction techniques which involved microwave-assisted extraction (MAE), aqueous two-phase extraction followed by microwave-assisted extraction (ATPE + MAE), and the "one-pot" microwave-assisted aqueous two-phase extraction (MA-ATPE) for extraction of solasonine and solamargine from leaves of $S$. mauritianum which was evaluated. The microwave-assisted extraction of solasonine and solamargine yielded optimums at $5.00 \mathrm{~min}$, microwave power of $270 \mathrm{~W}$, and solid/liquid of $0.1 \mathrm{~g} \mathrm{~L}^{-1}$ at an ethanol concentration of $60 \%$. Application of a two-stage extraction (MAE + ATPE) in CaO-dried alcohol resulted in decreased amounts of solasonine and solamargine extracted. The best yields of solasonine and solamargine were achieved in the MA-ATPE method. Extraction of solamargine and solasonine using $\mathrm{Na}_{2} \mathrm{CO}_{3}$ in $\mathrm{CaO}$-dried ethanol during MA-ATPE was approximately threefold and twofold greater than that of MAE + ATPE, respectively. Furthermore, extraction of solamargine and solasonine using $\mathrm{NaCl}$ in $\mathrm{CaO}-\mathrm{dried}$ ethanol during MA-ATPE was approximately twofold greater than that of MAE + ATPE. The synergy of microwaves and salting-out in the "one-pot" MA-ATPE technique was shown to be a contributing factor for enhanced extraction of solamargine and solasonine from leaves of $S$. mauritianum. Application of this time- and energy-efficient extraction method could potentially be expanded for enrichment of medicinally important compounds from biomass of other medicinal plants.
\end{abstract}

Keywords Microwave-assisted extraction · Toxic phytochemicals · Solanum mauritianum $\cdot$ Aqueous two-phase extraction

\section{Introduction}

Plant reaction to damage is an inherent character and occurs through exhibition of defense mechanisms against herbivores and piercing-sucking insects such as whiteflies and bacterial pathogens [1-3]. One of the modes of operation of bacterial

Tebogo Mphatlalala Mokgehle

tebogo.mokgehle24@yahoo.com

1 Department of Chemistry, School of Mathematical and Natural Sciences, University of Venda, Private Bag X5050, Thohoyandou 0950, South Africa

2 Department of Biochemistry, School of Mathematical and Natural Sciences, University of Venda, Private Bag X5050, Thohoyandou 0950, South Africa

3 Department of Ecology and Resource Management, School of Environmental Sciences, University of Venda, Private Bag X5050, Thohoyandou 0950, South Africa pathogens involves the release of reactive oxygen species such as singlet oxygen and peroxide radicals which result in oxidative stress leading to cellular damage in the host plants [1]. Other anti-pathogenic modes of plant include inhibition of glioma growth [4] and apoptosis of human chordoma cells [5]. Defense mechanisms against biotic stressors also involve the accumulation of toxic secondary metabolites, such as alkaloids which directly reduce the fitness of the invader $[3,6,7]$. One special class of alkaloids are steroidal glycoalkaloids found in numerous members of the Solanaceae family. They are composed of nitrogen-containing alkaloid groups and carbohydrate sugar side chains [3]. Extraction of toxic phytochemicals from Solanum plants is essential as they are the richest bioresource of drugs for medicinal applications [6]. Glycoalkaloids have been known for their pharmacological effectiveness towards human health such as being antidiabetic [8], antifungal [9], antiparasitic [10], 
and anticancer [11]. Hence, extraction of these invaluable naturally derived compounds (glycoalkaloids) is worthwhile.

Microwave-assisted extraction (MAE) is a simple environmentally friendly and economical technique for the extraction of biologically active compounds from different plant materials $[12,13]$. The advantage of this technique includes shorter extraction time, lesser solvent requirement, improved purity of the extract, low cost, and better extraction yield in comparison to Soxhlet extraction. This extraction method is a quick and highly effective technique for obtaining extracts under mild conditions; therefore, it has been considered a potential alternative to traditional methods [14-16]. Aqueous two-phase extraction (ATPE) is desired for its environmental compatibility, low interfacial tension of phases, high yields, and low process time [17, 18]. Recently, researchers have turned their attention to an improved version of ATPE, salting-out assisted liquid-liquid extraction (SALLE) technique, which facilitates extraction of metabolites from complex matrices [19-21].

Glycoalkaloids are useful to a certain extent, since they have been shown to possess some bioactive properties which include anti-pesticide or anti-cancer activity [10, 11]. Despite the bioactive properties of glycoalkaloids, they are mainly toxic [3]. This work also aimed to explore conventional extraction methods such as MAE, aqueous twophase extraction followed by microwave-assisted extraction (MAE + ATPE) and microwave-assisted aqueous two-phase extraction (MA-ATPE) for enrichment of glycoalkaloids from Solanum mauritianum. To the best of our knowledge, S. mauritianum has been underexplored with regard to its metabolites. Though known to be an invasive weed species from the Solanaceae family, the plant has been studied to be an essential ingredient for South African traditional medicine for treatment of menorrhagia [22] dysentery, diarrhea [23], and infertility [24] due to its metabolite composition, which is comprised of a bioactive class of compounds, glycoalkaloids. Hence, the need arises to obtain these medicinally important glycoalkaloid metabolites such as solasonine and solamargine from $S$. mauritianum using environmentally friendly extraction methods. Ethanol was used as an extractant because it can be regarded as a green solvent [25-28], because it is a nontoxic solvent. The application of microwaves or aqueous two-phase systems in conjunction with ethanol could potentially pave the way for more reliable means of obtaining these compounds and its sustained use in metabolomics. Furthermore, application of plant-based phytocompounds in medicine could likely eliminate the need for metabolic compounds synthesized in the lab, of which are often laborious and expensive.

\section{Materials and methods}

\subsection{Sample collection}

The leaves of S. mauritianum were obtained from Phiphidi, Limpopo, in October 2019, South Africa. The plants were air-dried until a constant weight was obtained, and the leaves were ground with a rotating blade blender into a fine powder with particle sizes ranging from 100 to $300 \mu \mathrm{m}$. Thereafter, this powder was stored in glass containers and covered to prevent light penetration.

\subsection{Chemicals and materials}

Absolute ethanol (99.9\% CP), which was used as an extraction solvent, was purchased from Associated Chemical Enterprises (Johannesburg, South Africa). Ultra-pure water $(0.005 \mu \mathrm{s}, 18 \mathrm{~m} \Omega)$ was used for preparation of ethanol solutions. A modified microwave oven (DM 350, Defy, Polokwane, South Africa) of 28 L capacity, working at a frequency of $2450 \mathrm{MHz}$, was used for microwave-assisted extraction. The salts for ATPE $\mathrm{NaCl}$ (anhydrous $>99 \%$ purity) and $\mathrm{Na}_{2} \mathrm{CO}_{3}$ (anhydrous $>99 \%$ purity) and the drying agent $\mathrm{CaO}$ (reagent grade $>99 \%$ purity) were all purchased from Associated Chemical Enterprises (Johannesburg, South Africa). Whatman grade 1 filter papers were purchased from Sigma-Aldrich (Johannesburg, South Africa).

\subsection{Extraction procedure}

\subsubsection{MAE}

Ground leaves of S. mauritianum plant powder (mass: 0.6-1.4 g) was immersed in hydroalcoholic solutions with various concentrations $(20-60 \%)$ contained in a 1 -necked $250-\mathrm{mL}$ round-bottomed flask at irradiation time (1-13 min) with power varied from 90 to $900 \mathrm{~W}$. The method is illustrated in Appendix (Fig. 8a).

\subsection{2 $\mathrm{MAE}+\mathrm{ATPE}$}

The optimized parameters following chromatographic analysis (details are included in Sect. 2.4) for the extraction of solasonine and solamargine during MAE was a $60 \%$ ethanol extraction solvent, irradiation time of $5 \mathrm{~min}$ at a power of $270 \mathrm{~W}$. A ground S. mauritinum biomass powder with a mass of $1.0 \mathrm{~g}$ was immersed in $30 \%(\mathrm{w} / \mathrm{v})$ of $\mathrm{NaCl}$ (chaotrope) or $\mathrm{Na}_{2} \mathrm{CO}_{3}$ (kosmotrope). Thereafter, the extract $(5 \mathrm{~mL})$ 
obtained from the optimized conditions during MAE was mixed with either $30 \%(\mathrm{w} / \mathrm{v})$ of $\mathrm{NaCl}(5 \mathrm{~mL})$ or $30 \%(\mathrm{w} / \mathrm{v})$ of $\mathrm{Na}_{2} \mathrm{CO}_{3}(5 \mathrm{~mL})$ followed by the addition of the extraction solvents- $\mathrm{CaO}$-dried ethanol or $99 \%$ ethanol $(10 \mathrm{~mL})$ - which resulted in an aqueous two-phase system. The spontaneous formation of ATPE, consisting of a lower aqueous phase and an upper ethanolic phase, under the conditions stated above was also reported by Mokgehle et al. [3]. Hence, this extraction was a sum of two solutions, MAE followed by ATPE. $\mathrm{CaO}$-dried ethanol was prepared by adding $25 \mathrm{~g}$ of $\mathrm{CaO}$ drying agent to $100 \mathrm{~mL}$ of $99 \%$ ethanol. The mixture was stirred for 10 min before filtration with a Whatman grade 1 filter paper. The method is illustrated in Appendix (Fig. 8b).

\subsubsection{MA-ATPE}

In this "one-pot" extraction, the optimized results from MAE were applied on the ATPE solution which consisted of ground plant powder mass: $1 \mathrm{~g}$, saturated salt of $30 \%(\mathrm{w} / \mathrm{v})$ of $\mathrm{NaCl}$ (chaotrope) or $\mathrm{Na}_{2} \mathrm{CO}_{3}$ (kosmotrope) which formed the bottom phase while the upper phase extraction solvent consisted of CaO-dried or $99 \%$ ethanol $(10 \mathrm{~mL})$. In MAATPE, both the saturated salt solution 30\% (w/v), consisting of either $\mathrm{NaCl}$ or $\mathrm{Na}_{2} \mathrm{CO}_{3}$, and the ethanol extraction solvent were contained in the round-bottomed flask, all in the microwave oven. The spontaneous formation of ATPE under the conditions stated above was also reported by Mokgehle et al. [3]. In all the three extraction methods conducted, there was no agitation of the sample solution prior to or following extraction. Furthermore, the volumes of the solvents after the extraction period remained unaltered. The setup of the MA-ATPE systems is shown in Appendix (Fig. 8c).

\subsection{Analysis on the UHPLC-qTOF-MS}

Chromatographic separation of the extracts (upper ethanolic phase) obtained from the ATPE mixture was conducted on a UHPLC-qTOF-MS 9030 mass spectrometer (Shimadzu, Japan). This instrument contained a Shimpack $\mathrm{C}_{18}$, $2.1 \times 100 \mathrm{~mm}, 2.7-\mu \mathrm{m}$ column from Shimadzu (Honeydew, South Africa) where the mobile phase consisted of formic acid $(0.1 \%)$ in deionized water (solvent $\mathrm{A}$ ) and methanol with $0.1 \%$ formic acid (solvent B). Chromatographic separation was achieved using a 30-min gradient elution method consisting of the following settings: the initial conditions were $5 \%$ solvent $\mathrm{B}$ at a flow rate of $0.4 \mathrm{~mL} \mathrm{~min}^{-1}$ and held constant for $3 \mathrm{~min}$. Conditions were then changed to $45 \%$ solvent B at 9 min, increased slightly to $50 \%$ solvent $A$ at $21 \mathrm{~min}$, and then quickly ramped up to $90 \%$ solvent $\mathrm{B}$ at $22 \mathrm{~min}$ and kept constant for $3 \mathrm{~min}$. Conditions were changed to $5 \%$ solvent $\mathrm{B}$ at $27 \mathrm{~min}$ and kept for $3 \mathrm{~min}$ to allow for re-equilibration before the next run.

For mass spectrometry, the acquisition parameters as discussed by Ramabulana et al. [29] were used. Briefly, MS data were acquired using positive electron spray ionization (ESI) modes. The MS was configured to scan the range of 100-1000 Da with a scan time of $0.2 \mathrm{~s}$. After a series of optimization, the following settings were found to be optimal: capillary voltage of $4.5 \mathrm{eV}$, sample cone potential of $30 \mathrm{~V}$, source temperature of $120^{\circ} \mathrm{C}$, desolvation temperature of $450{ }^{\circ} \mathrm{C}$, desolvation gas flow of $550 \mathrm{~L} \mathrm{~h}^{-1}$, and multichannel plate detector potential of $1600 \mathrm{~V}$. In order to achieve efficient fragmentation to aid during identification, the mass spectrometry data were collected using a collision energy ramp of 10-30 eV and, when necessary, a higher collision energy ramp of 60-165 eV was also used. Structural elucidation was done using KNapSAck online metabolite database. Chemical identification was done using KNapSAck Core System online metabolite database (version 1.200.03) [30].

\section{Results and discussion}

\subsection{Chromatographic profile of solasonine and solamargine}

In Fig. 1, the mass spectrometry and elution profile of two glycoalkaloid isomers are shown, where Fig. 1a indicates solasonine $m / z, 884$ and b solamargine $m / z$ 868. Both contain the same aglycone unit solasodine, yet only differ in monosaccharides glycosylated to the aglycone unit. For instance, solamargine contains two rhamnose monosaccharides and glucose while solasonine is composed of glucose, rhamnose, and galactose, which account for the 16 mass unit difference between the two compounds (Fig. 1a and b). Figure 1c shows a 30-min base peak chromatogram of a $60 \%$ ethanolic MAE extract from $S$. mauritianum, which also shows the elution order of two glycoalkaloids with the relatively more polar solasonine eluting at $19.56 \mathrm{~min}$ and solamargine at $19.76 \mathrm{~min}$ from the reversed phase column. Furthermore, Munari et al. [31] observed a similar trend in the extracts of Solanum lycopersicum on as Zorbax SB-C18 column where solasonine eluted before solamargine which indicated the relatively higher polarity of the former. In another study, Chester et al. [32] quantified solasonine and solamargine obtained from extracts of Solanum nigrum L. based on retention factors $\left(\mathrm{R}_{\mathrm{f}}\right)$ on the HPTLC chromatogram on the UPLC-ESI-MS/MS. In the same work, solasonine had a lower $\mathrm{R}_{\mathrm{f}}$ than solamargine due to its relatively higher polarity. Hence, the presence of galactose is a major contributor 

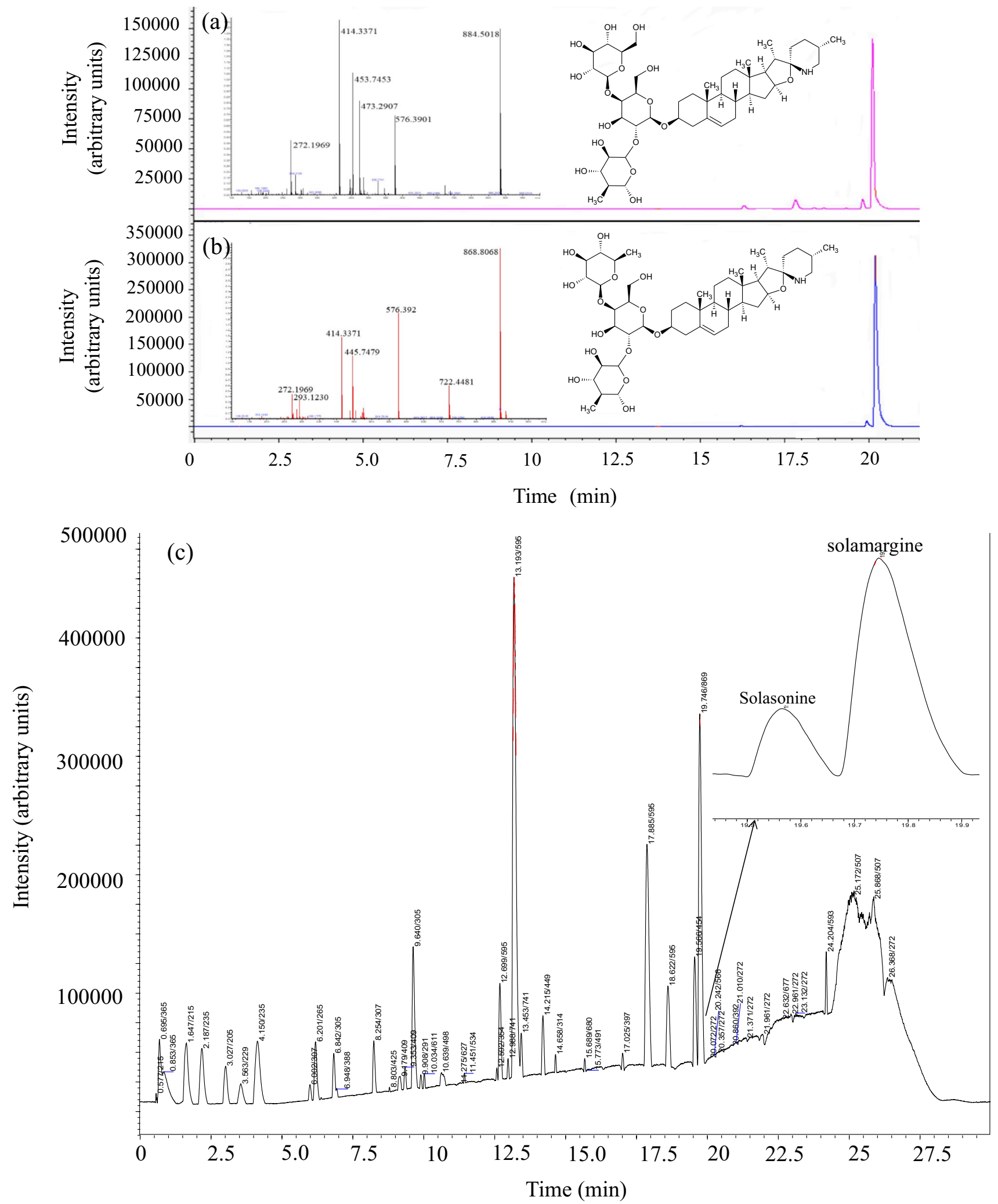

Fig. 1 Elution and mass spectrometry profile of two closely related glycoalkaloid isomers a solasonine $\left(t_{\mathrm{R}}(\min )-19.56\right)$ and $\mathbf{b}$ solamargine $\left(t_{\mathrm{R}}\right.$ (min) - 19.76). c 30-min base peak chromatogram (BPC) of a crude (60\% ethanolic extract) of $S$. mauritianum 
Fig. 2 Effect of (\%) ethanol for MAE of solasonine and solamargine. Conditions: irradiation time: $5 \mathrm{~min}$, power: $270 \mathrm{~W}$, mass of plant: $1.00 \mathrm{~g}(n=3$, $\mathrm{RSD})$

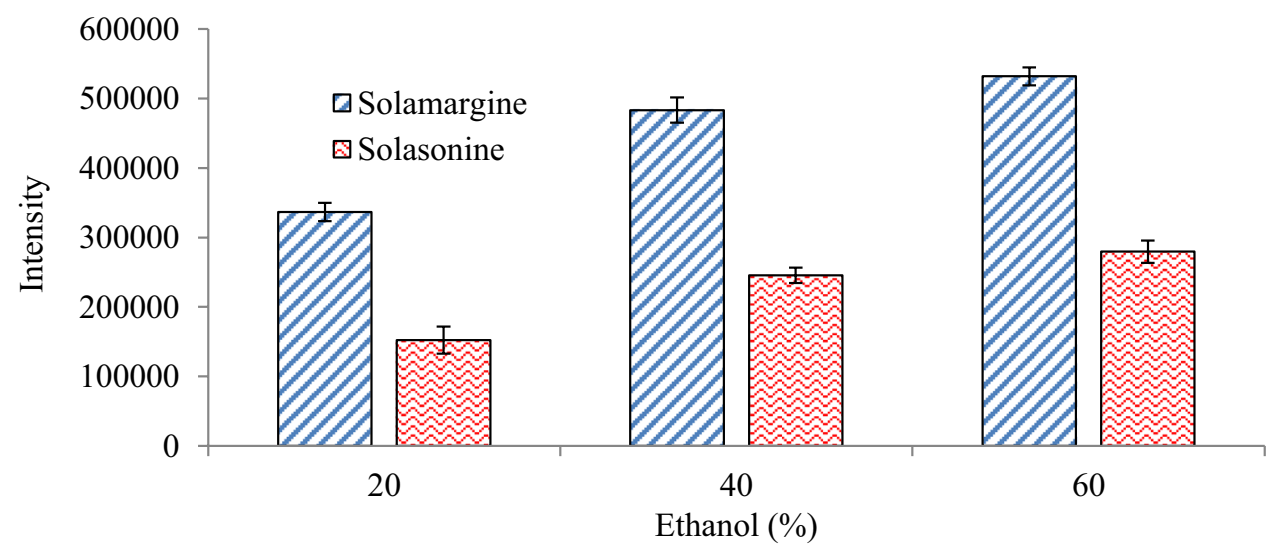

to the polarity of solasonine and its relatively higher affinity for the $\mathrm{CaO}$-dried ethanol extraction solvent.

\subsubsection{Effect of concentration of ethanol on MAE}

A study investigating the effect of ethanol percentage on the extraction of solasonine and solamargine was conducted as shown in Fig. 2. As the concentration of ethanol was increased, the intensities of both solasonine and solamargine also increased. The higher intensities which correlated with the higher yield can be attributed to the higher proportion of ethanol and the lower level of water in the extraction solvent. The ethanol percentage in water was one of the driving factors influencing the MAE of compounds as it affected the solubility of metabolites, penetration of solvent into the cells of plants, interaction of solvent with matrix, and the absorption of microwave energy [33]. Increasing the water concentration in the solvent as highlighted by Veggi et al. [34] and Zhang et al. [35] has been reported to influence selectivity during extraction, which resulted in a greater affinity towards proteins and carbohydrates rather than bioactive plant metabolites. It was also observed that the relative intensities of solamargine to solasonine occurred on a general ratio of $2: 1$, which probably indicated the greater relative abundance of solamargine compared to solasonine in the leaves of S. mauritianum. Hence, the optimum extraction solvent was $60 \%$ ethanol and was then used in the following study.
Fig. 3 Effect of solid/liquid ratio for MAE of solasonine and solamargine. Conditions: irradiation time: $5 \mathrm{~min}$, power: $270 \mathrm{~W}$, solvent: $60 \%$ ethanol $(n=3, \mathrm{RSD})$

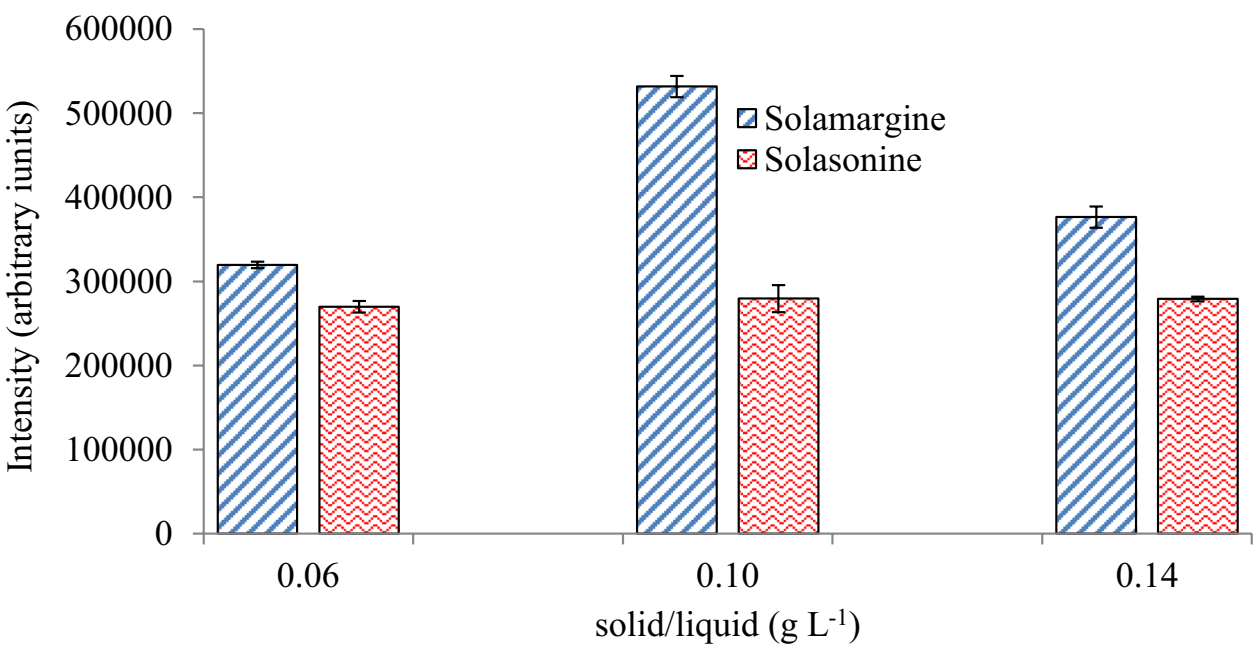




\subsubsection{Effect of solid/liquid ratio on MAE}

The effect of solid/liquid ratio was evaluated where $0.6,1$, and $1.4 \mathrm{~g}$ were evaluated in $10 \mathrm{~mL} 60 \%$ ethanol and corresponded to solid/liquid ratios of $0.06,0.1$, and $0.14 \mathrm{~g} \mathrm{~L}^{-1}$, respectively (Fig. 3). The optimal extraction of solasonine and solamargine was observed at $0.1 \mathrm{~g} \mathrm{~L}^{-1}$. The lower intensities of extracted solamargine and solasonine at $0.06 \mathrm{~g} \mathrm{~L}^{-1}$ could be due to the relatively lower amount of plant material used. Increased solvent volumes have also been reported to reduce the heating efficiency, limit the breakage of solid cell walls, and inhibit the driving force for the mass transfer of compounds [33]. Similarly, Alara et al. [36] reported that larger volumes of solvent required more energy and time to maximize extraction of analytes from the plant matrix. It was also noted that with larger solid/liquid ratios, reduced extraction of solasonine and solamargine was observed. This could be as a result of lumping of the powdered plant material, limiting access for the extraction solvent to penetrate through the cell walls. Similarly, Sajid et al. [37] reported on clogging during solid-phase micro-extraction. The optimum mass for extraction of solasonine and solamargine was $1 \mathrm{~g}$, with a solid/liquid ratio of $0.1 \mathrm{~g} \mathrm{~L}^{-1}$.

\subsubsection{Effect of irradiation time on MAE}

A study evaluating the effect of irradiation time on extraction of solasonine and solamargine was performed as shown in Fig. 4. As time was increased from 1 to $5 \mathrm{~min}$, a gradual increase in the extraction of solasonine and solamargine was noted. In general, higher extraction time tends to increase the yield of extraction. In addition, the dielectric properties of solvents used in MAE may have significant impacts on the extraction time $[34,38]$. For instance, at room temperature, water has a dielectric constant of 80 ; however, the addition of ethanol in the aqueous mixture reduces this constant, greatly allowing it to easily dissolve a wide range of less polar metabolites; hence, in this instance, it only took 5 min to achieve optimal extraction of both solasonine and solamargine, whereas if only water was used as an extractant, optimal extraction of both glycoalkaloids would have most likely taken longer than 5 min [34, 38]. Furthermore, extraction of metabolites from the plant matrix is not an
Fig. 4 The influence of irradiation time on the MAE of solasonine and solamargine. Conditions: mass of plant: 1.00 g, power: $270 \mathrm{~W}$, solvent: $60 \%$ ethanol $(n=3$; RSD)

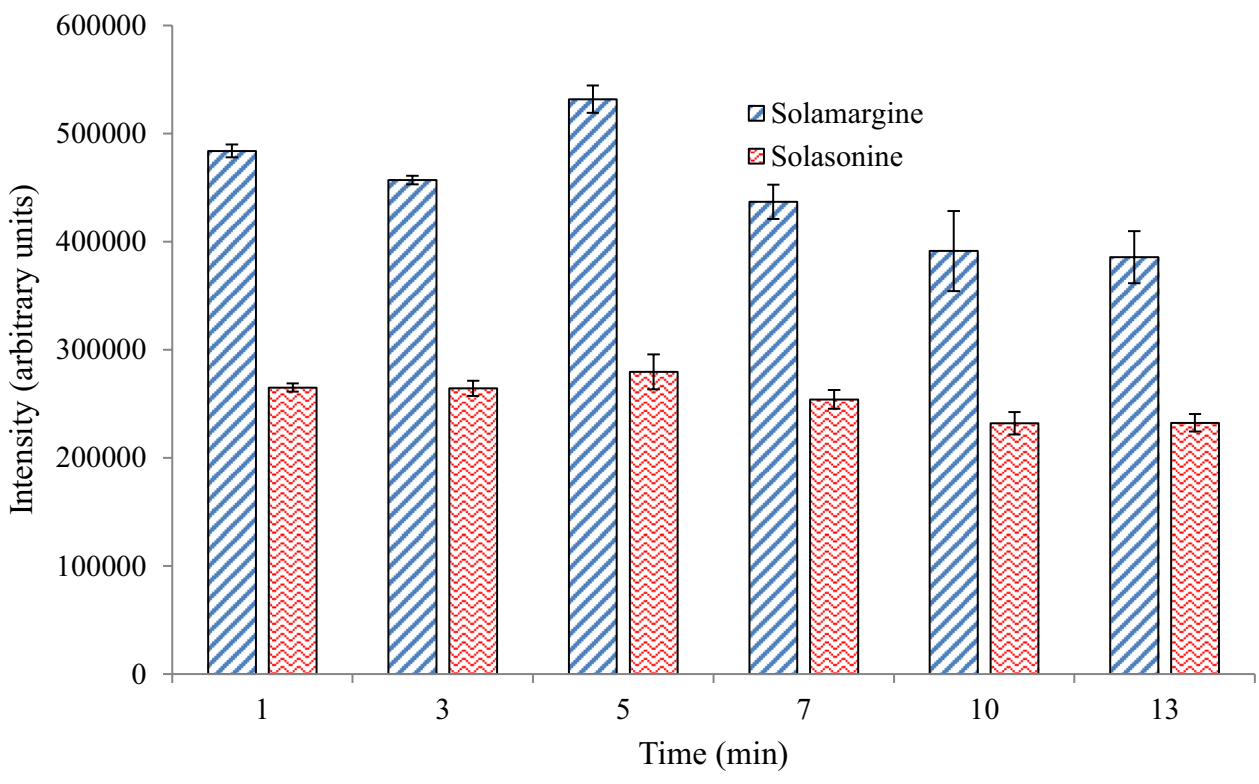


Fig. 5 Evaluation of the effect of microwave power on the MAE of solamargine and solasonine. Conditions: mass of plant: $1.00 \mathrm{~g}$, solvent: $60 \%$ ethanol, time: $5 \min (n=3, \mathrm{RSD})$

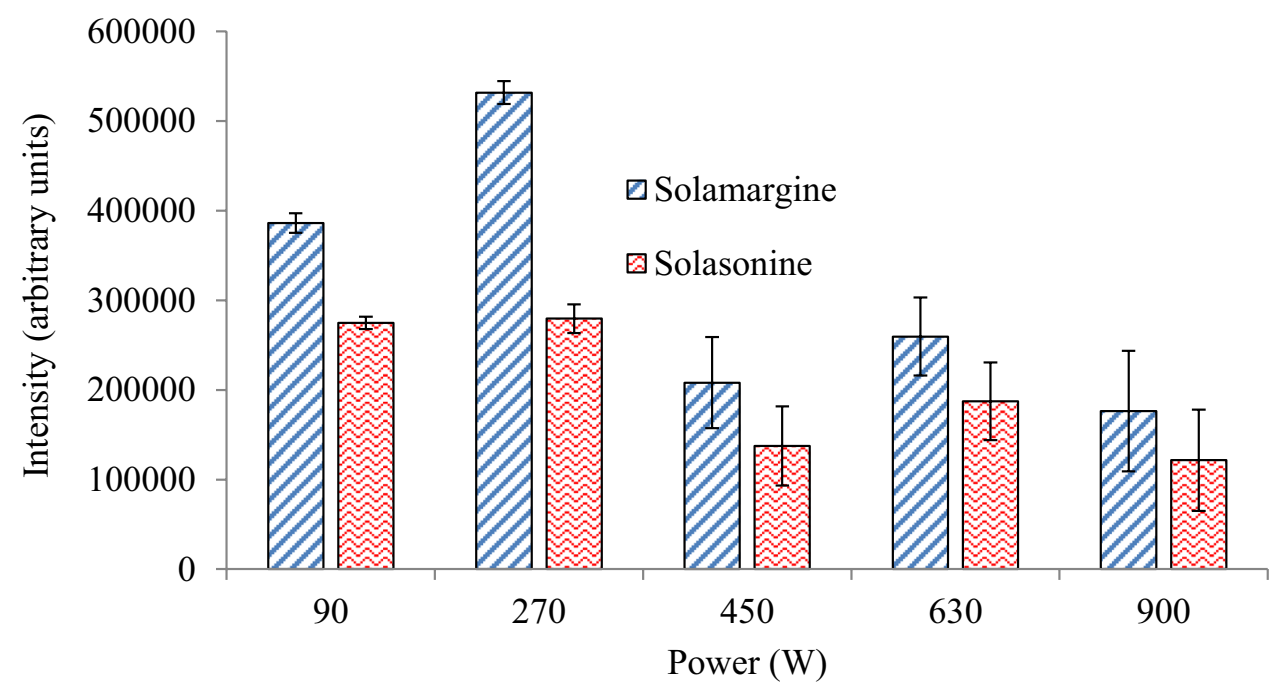

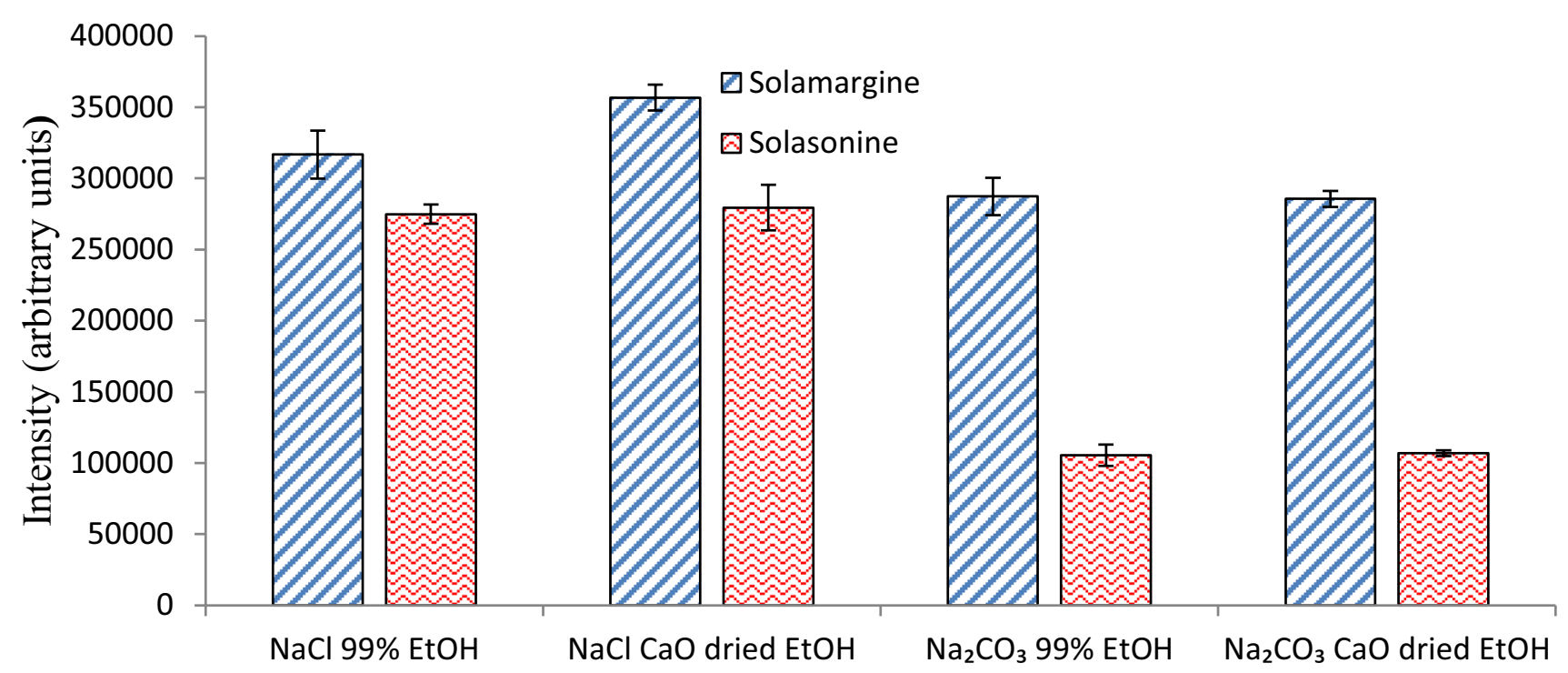

Fig. 6 Comparison on MAE + ATPE of solasonine and solamargine when 99\% absolute ethanol and absolute ethanol dried with CaO was used in the presence of the chaotrope $\mathrm{NaCl}$ and kosmotrope $\mathrm{Na}_{2} \mathrm{CO}_{3}(n=3, \mathrm{RSD})$

instantaneous process; there are multiple phases occurring which involve removal of compounds from the outer surface of plant matrix, a transition state consisting of intermolecular forces between the metabolites and the plant matrix inhibiting mass transfer brought about by the extraction solvent. After the 5-min irradiation time, a steady decrease in the intensities of both glycoalkaloids was observed (Fig. 4). This steady decrease at longer times was increased which could be associated with the increased degradation of thermolabile metabolites, solasonine and solamargine, during this period. This is in agreement with what was reported by Doulabi et al. [33] and Veggi et al. [34]. The optimal extraction time to be used in the following studies was $5 \mathrm{~min}$.

\subsubsection{Effect of power (W) on MAE}

Power studies were conducted to examine its effect on the extraction of solasonine and solamargine as shown in Fig. 5. 


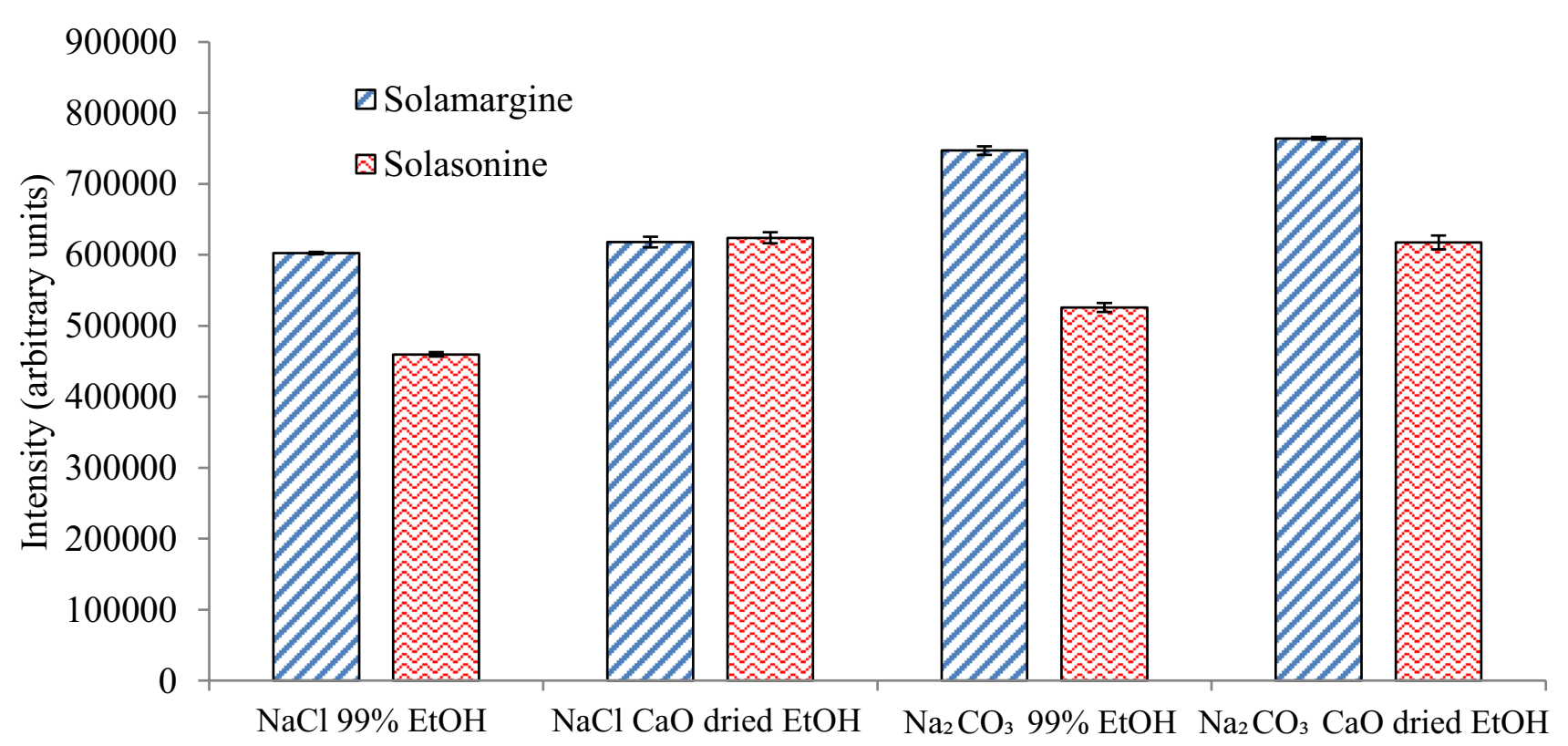

Fig. 7 Comparative MA-ATPE of solasonine and solamargine when 99\% ethanol and ethanol dried with $\mathrm{CaO}$ were used in the presence of the chaotrope $\mathrm{NaCl}$ and kosmotrope $\mathrm{Na}_{2} \mathrm{CO}_{3}(n=3, \mathrm{RSD})$

From 90 to $270 \mathrm{~W}$, an increase in extraction of both glycoalkaloids was observed (Fig. 5). Generally, an increase in microwave power can improve the penetration of solvent into plant matrix, resulting in rapid delivery of microwave energy to both solvent and plant matrix [33]. Accordingly, from 90to $270-\mathrm{W}$ microwave power, dissolution of solasonine and solamargine occurred due to the increased temperature of the extraction solvent. However, from 270 to $900 \mathrm{~W}$, a steady decrease was observed (Fig. 5). This could be because of excessive microwave irradiation energy degrading both solasonine and solamargine [38, 39]. Therefore, the optimal extraction power was observed at $270 \mathrm{~W}$.

\subsection{MAE + ATPE}

Following the optimized result obtained for MAE (Sect. 3.1) which included $60 \%$ ethanol extraction solvent, solid/ liquid ratio of $0.1 \mathrm{~g} \mathrm{~L}^{-1}$, irradiation time of $5 \mathrm{~min}$, and power of $270 \mathrm{~W}$, the obtained conditions were applied for MAE + ATPE to improve the extraction of solasonine and solamargine. Besides the inclusion of ATPE, the 99\% ethanol extraction solvent was dried with $\mathrm{CaO}$ drying agent with the aim of enhancing the extraction of solasonine and solamargine. Figure 6 shows the extraction profile for solasonine and solamargine using an extraction solvent of $99 \%$ ethanol and $\mathrm{CaO}-$ dried ethanol when the chaotrope and kosmotrope $\mathrm{NaCl}$ and $\mathrm{Na}_{2} \mathrm{CO}_{3}$ were used, respectively. In general, no differences were observed in intensities of solasonine and solamargine when $\mathrm{CaO}$-dried ethanol was used as an extraction solvent compared to $99 \%$ ethanol for both $\mathrm{Na}_{2} \mathrm{CO}_{3}$ and $\mathrm{NaCl}$ (Fig. 6). Though $\mathrm{CaO}$ was reported as an efficient drying agent by Danish et al. [40] and Jia et al. [41] in sewage sludge and mortar, respectively, its application in drying
Table 1 Intensities of solasonine and solamargine under MAE, MAE + ATPE, and MA-ATPE

\begin{tabular}{|c|c|c|c|c|c|}
\hline & \multirow[t]{3}{*}{ Salt } & \multicolumn{4}{|c|}{ Intensity (arbitrary units) } \\
\hline & & \multicolumn{2}{|l|}{ Solasonine } & \multicolumn{2}{|l|}{ Solamargine } \\
\hline & & $\mathrm{CaO}$-dried $\mathrm{EtOH}$ & EtOH $99.9 \%$ & $\mathrm{CaO}$-dried $\mathrm{EtOH}$ & EtOH $99.9 \%$ \\
\hline MAE & - & - & 279,507 & - & 531,704 \\
\hline \multirow[t]{2}{*}{ MAE + ATPE } & $\mathrm{NaCl}$ & 279,507 & 274,890 & 356,772 & 316,784 \\
\hline & $\mathrm{Na}_{2} \mathrm{CO}_{3}$ & 106,899 & 105,495 & 285,572 & 287,307 \\
\hline \multirow[t]{2}{*}{ MA-ATPE } & $\mathrm{NaCl}$ & 623,912 & 459,642 & 618,115 & 602,356 \\
\hline & $\mathrm{Na}_{2} \mathrm{CO}_{3}$ & 617,550 & 525,691 & 763,974 & 746,758 \\
\hline
\end{tabular}


extraction solvents for the improving extraction of solasonine and solamargine was limited.

\subsection{MA-ATPE}

With the aim of further improving the extraction of solamargine and solasonine from $S$. mauritianum, a hyphenated MA-ATPE was applied as shown in Fig. 7. In both instances where the extraction solvents were $99 \%$ ethanol and $\mathrm{CaO}$ dried ethanol, $\mathrm{Na}_{2} \mathrm{CO}_{3}$ was generally observed to be a better extractant of solasonine and solamargine than $\mathrm{NaCl}$. The opposite effect was observed for the two-stage extraction process MAE + ATPE (Fig. 6), which indicated that the extraction method influenced the salting-out efficiency of solamargine and solasonine from the aqueous solution in the presence of $\mathrm{NaCl}$ or $\mathrm{Na}_{2} \mathrm{CO}_{3}$. The effect of extraction conditions on salting-out was also reported by Tajeda-Casado et al. [20] and Sazali et al. [21]. The doubly charged carbonate ions from $\mathrm{Na}_{2} \mathrm{CO}_{3}$ interacted with the hydration sphere surrounding the solute (solamargine and solasonine) to a greater extent than singly charged chloride ions from $\mathrm{NaCl}$, forming carbonic acid. Thereafter, this led to the precipitation (salting-out) of the solute in the aqueous phase and resultant extraction by ethanol. Salting-out effect has been reported to aid the extraction of vitamin D3 from milk samples [21] and in the determination of 5-nitroimidazolesin in fish [19].

\subsection{Comparison of the extraction efficiency of MAE, MAE + ATPE, and MA-ATPE}

Enrichment of solasonine and solamargine was considerably greater for MA-ATPE in comparison to MAE + ATPE and MAE. For instance, extraction of solamargine and solasonine using $\mathrm{Na}_{2} \mathrm{CO}_{3}$ in $\mathrm{CaO}$-dried ethanol during MA-ATPE (Fig. 7) was approximately threefold and twofold greater than that of MAE + ATPE, respectively (Fig. 6). Similarly, extraction of solamargine and solasonine using $\mathrm{NaCl}$ in CaO-dried ethanol during MA-ATPE (Fig. 7) was approximately twofold greater than that of MAE + ATPE (Fig. 6, Table 1). This indicated that in MA-ATPE, the synergistic effect of microwaves and salting-out occurred simultaneously. In this case, extraction was assisted by microwaves which resulted in cell wall rapture and subsequent mass transfer of solasonine and solamargine into the aqueous solution. In addition to microwaves, the salting-out effect prompted the precipitation of solasonine and solamargine from the hydration sphere into the ethanol extraction phase during MA-ATPE. The two-step extraction method MAE + ATPE was observed to have the lowest extraction of metabolites (Fig. 6). This could be attributed to the interstep loss of metabolites during MAE-ATPE. Furthermore,
Gardernar et al. [42] highlighted some disadvantages associated with two-step extractions which involved the requirement for a substantial amount of specimen to prevent analyte loses. It was also observed that MA-ATPE improved extraction of solasonine and solamargine compared to MAE (Table 1). This suggested that the chaotrope and kosmotrope were influential in aiding extraction of solasonine and solamargine in MA-ATPE compared to MAE, from which the extraction did not include salts. Hence, the simultaneous application of microwaves assisted the extraction of solasonine and solamargine in the aqueous two-phase system based on salting-out, as seen with MA-ATPE, and was influential in enhancing the extraction of solasonine and solamargine in comparison to MAE and MAE + ATPE.

\section{Conclusions}

In this study, the optimization of microwave and aqueous two-phase-based extraction techniques which involved MAE, ATPE + MAE, and MA-ATPE for extraction of solasonine and solamargine from leaves of $S$. mauritianum was evaluated. The first technique which only involved microwaves, MAE, maximal extraction of solasonine and solamargine was achieved when extraction was conducted for $5 \mathrm{~min}$, microwave power of $270 \mathrm{~W}$, solid/liquid of $0.1 \mathrm{~g} \mathrm{~L}^{-1}$, and an ethanol concentration of $60 \%$. Efforts were then made to improve extraction of both solasonine and solamargine by applying drying agents such as $\mathrm{CaO}$ in the ethanol extraction solvent. Dried ethanol was applied in two-stage extraction (MAE + ATPE). However, the yields of solasonine and solamargine were observed to decrease due to possible analyte loss of metabolites during inter-step transfer between MAE and ATPE. Application of CaO-dried ethanol in conjunction with the "one-pot" MA-ATPE was shown to considerably enhance extraction of both glycoalkaloids relative to MAE and MAE-ATPE. For instance, extraction of solamargine and solasonine using $\mathrm{Na}_{2} \mathrm{CO}_{3}$ in $\mathrm{CaO}$-dried ethanol during MAATPE was approximately threefold and twofold greater than that of MAE + ATPE, respectively. Furthermore, extraction of solamargine and solasonine using $\mathrm{NaCl}$ in $\mathrm{CaO}$-dried ethanol during MA-ATPE was approximately twofold greater than that of MAE+ ATPE. Hence, the kosmotrope $\left(\mathrm{Na}_{2} \mathrm{CO}_{3}\right)$ was shown to be a relatively better extractor of solamargine and solasonine in comparison to the chaotrope $(\mathrm{NaCl})$ due to its superior salting-out capacity in MA-ATPE. The results suggested that MA-ATPE, a technique propelled by the synergy of microwaves and salting-out, is a promising time- and energy-efficient method for enrichment of solamargine and solasonine from leaves of $S$. mauritianum. 


\section{Appendix}

Fig. 8 Experimental setup of (a) MAE, (b) MAE + ATPE, and (c) MA-ATPE
Acknowledgements The authors would like to express their gratitude the University of Venda.

Author contribution TMM, NTT, and NEM conceived the study; TMM and NEM conducted the experiments and data analyses. NTT, NEM, and WMG supervised the project. WMG helped to draft the manuscript. All authors read and approved the final manuscript.

Funding This study received financial support from the National Research Foundation and Sasol Inzalo.

Data availability (data transparency) Not applicable.

Code availability (software application or custom code) Not applicable.

\section{Declarations}

Competing interests The authors declare no competing interests. (b) MAE+ATPE

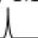

a) MAE

$\mathrm{MAE}$

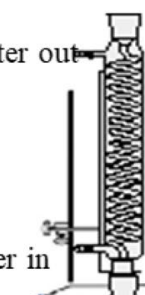

k

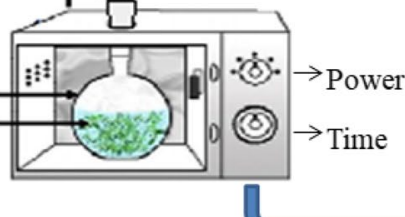

Hydrophobic compounds

- Hydrophilic compounds

$99 \%$ EtOH with drying agents $\mathrm{CaO}$

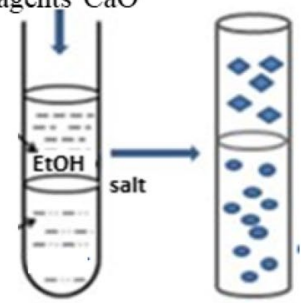

MAE

mixture

(c) MA-ATPE

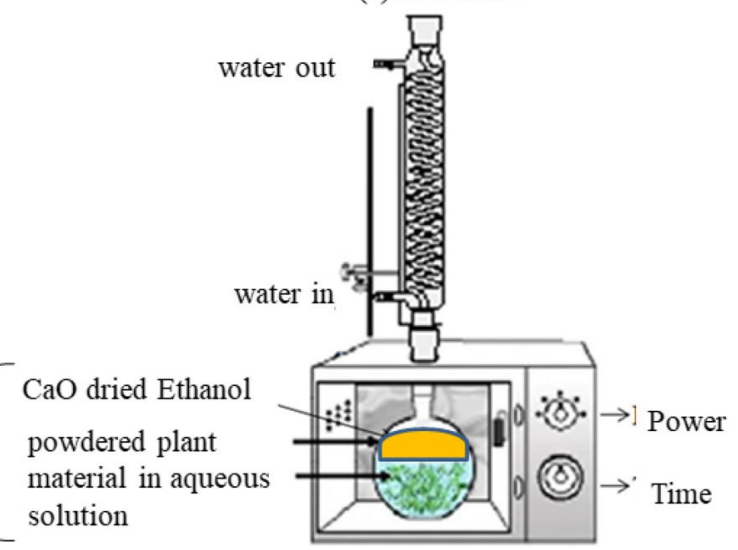

Open Access This article is licensed under a Creative Commons Attribution 4.0 International License, which permits use, sharing, adaptation, distribution and reproduction in any medium or format, as long as you give appropriate credit to the original author(s) and the source, provide a link to the Creative Commons licence, and indicate if changes were made. The images or other third party material in this article are included in the article's Creative Commons licence, unless indicated otherwise in a credit line to the material. If material is not included in the article's Creative Commons licence and your intended use is not permitted by statutory regulation or exceeds the permitted use, you will need to obtain permission directly from the copyright holder. To view a copy of this licence, visit http://creativecommons.org/licenses/by/4.0/.

\section{References}

1. Santamaria ME, Martínez M, Cambra I, Grbic V, Diaz I (2013) Understanding plant defence responses against herbivore attacks: an essential first step towards the development of sustainable 
resistance against pests. Transgenic Res 22:697-708. https://doi. org/10.1007/s11248-013-9725-4

2. Montero-Vargus JM, Casarrubias-Castillo K, Martinez-Gallardo N, Ordaz-Ortiz JJ, Delano-Frier JP, Winkle R (2018) Modulation of steroidal glycoalkaloid biosynthesis in (Solanum lycoperium) by jasmonic acid. Plant Sci 277:155-165. https://doi.org/10. 1016/j.plantsci.2018.08.020

3. Mokgehle TM, Madala N, Gitari WM, Tavengwa NT (2021) Advances in the development of biopolymeric adsorbents for the extraction of metabolites from nutraceuticals with emphasis on Solanaceae and subsequent pharmacological applications. Carbohydr Polym 264:1-10. https://doi.org/10.1016/j.carbpol.2021. 118049

4. Wang X, Zou S, Lan YL, Xing JS, Lan XQ, Zhang B (2017) Solasonine inhibits glioma growth through anti-inflammatory pathways. Am J Transl Res 9:3977-3989

5. Zhao L, Wang L, Di SN, Xu Q, Ren QC, Chen SZ, Huang N, Jia D, Shen XF (2018) Steroidal alkaloid solanine A from Solanum nigrum Linn. exhibits anti-inflammatory activity in lipopolysaccharide/interferon $\gamma$-activated murine macrophages and animal models of inflammation. Biomed Pharmacother 105:606-615. https://doi.org/10.1016/j.biopha.2018.06.019

6. Baldwin IT, Schmelz EA, Ohnmeiss TE (1994) Wound-induced changes in root and shoot jasmonic acid pools correlate with induced nicotine synthesis in Nicotiana sylvestris spegazzini and comes. J Chem Ecol 20:2139-2157. https://doi.org/10.1007/ BF02066250

7. Rodsamran P, Sothornvit R (2019) Microwave heating extraction of pectin from lime peel: characterization and properties compared with the conventional heating method. Food Chem 278:364-372. https://doi.org/10.1016/j.foodchem.2018.11.067

8. Al-Ashaala HA, Farghaly AA, Abdel-Samee NS (2018) Antidiabetic efficacy of Solanum torvum extract and glycoalkaloids against diabetes induced mutation in experimental animals. Int $\mathbf{J}$ Pharm Sci Res 10:1323-1331

9. Grillo G, Gunjević V, Radošević K, Radojčić Redovniković I, Cravotto G (2020) Deep eutectic solvents and nonconventional technologies for blueberry-peel extraction: kinetics, anthocyanin stability, and antiproliferative activity. Antioxidants 9:1069-1097. https://doi.org/10.3390/antiox9111069

10. Anwar A, Mungroo MR, Khan S, Fatima I, Rafique R, Khan KM, Siddiqui R, Khan NA (2020) Novel azoles as antiparasitic remedies against brain-eating amoebae. Antibiotics 9:1-13. https:// doi.org/10.3390/antibiotics9040188

11. Albarri R, Toprakçı I, Kurtulbaş E, Şahin S (2021) Estimation of diffusion and mass transfer coefficients for the microwave-assisted extraction of bioactive substances from Moringa oleifera leaves. Biomass Convers Biorefin 221:1-8. https://doi.org/10.1007/ s13399-021-01443-8

12. Li J, Qi H, Wang Y, Su Q, Wu S, Wu L (2016) Hollow fibre-stir bar sorptive extraction and microwave assisted derivatization of amino acids in biological matrices. J Chromatogr A 1474:32-39. https://doi.org/10.1016/j.chroma.2016.10.079

13. Aeenehvand S, Toudehrousta Z, Kamankesh M, Mashayekh M, Tavakoli HR, Mohammadi A (2016) Evaluation and application of microwave-assisted extraction and dispersive liquid liquid microextraction followed by high performance liquid chromatography for the determination of polar heterocyclic aromatic amines in hamburger patties. Food Chem 190:429-435. https://doi.org/10. 1016/j.foodchem.2015.05.103

14. Ahuja S, Diehl D (2006) Sampling and sample preparation. Oxford: Compr Anal Chem 47:15-40. https://doi.org/10.1016/ S0166-526X(06)47002-1

15. Barba FJ, Grimi N, Vorobiev E (2015) New approaches for the use of non-conventional cell-disruption technologies to extract potential food additives and nutraceuticals from microalgae. Food Eng Rev 7:45-62. https://doi.org/10.1007/s12393-014-9095-6

16. Castejon N, Luna P, Senorans FJ (2018) Alternative oil extraction methods from Echium plantaginium L. seeds using advanced techniques and green solvents. Food Chem 244:75-82. https://doi. org/10.1016/j.foodchem.2017.10.014

17. Xavier L, Freire MS, Vidal-Tato I, González-Álvarez J (2014) Aqueous two-phase systems for the extraction of phenolic compounds from eucalyptus (Eucalyptus globulus) wood industrial wastes. J Chem Technol Biotechnol 89:1772-1778. https://doi. org/10.1002/jctb. 4260

18. Iqbal M, Tao Y, Xie S, Zhu Y, Chen D, Wang X, Huang L, Peng D, Sattar A, Shabbir MAB, Hussain HF, Ahmed S, Yuan Z (2016) Aqueous two phase system (ATPS): an overview and advances in its applications. Biol Proced Online 18:1-18. https://doi.org/10. 1186/s12575-016-0048-8

19. Hernández-Mesa M, Cruces-Blanco C, García-Campaña AM (2018) Simple and rapid determination of 5-nitroimidazoles and metabolites in fish roe samples by salting-out assisted liquid-liquid extraction and UHPLC-MS/MS. Food Chem 252:294-302. https://doi.org/10.1016/j.foodchem.2018.01.101

20. Tejada-Casado C, Lara FJ, García-Campaña AM, del Olmo-Iruela M (2018) Ultra-high performance liquid chromatography with fluorescence detection following salting-out assisted liquid-liquid extraction for the analysis of benzimidazole residues in farm fish samples. J Chromatogr A 1543:58-66. https://doi.org/10.1016/j. chroma.2018.02.042

21. Sazali NH, Alshishani A, Saad B, Chew KY, Chong MM, Miskam M (2019) Salting out assisted liquid liquid extraction coupled with high performance liquid chromatography for the determination of vitamin D3 in milk samples. R Soc Open Sci 6:1-8. https://doi. org/10.1098/rsos.190952

22. Uche-Okereafor N, Sebola T, Tapfuma K, Mekuto L, Green E, Mavumengwana V (2019) Antibacterial activities of crude secondary metabolite extracts from Pantoea species obtained from the stem of Solanum mauritianum and their effects on two cancer cell lines. Int J Environ Res Public Health 16:1-12. https://doi. org/10.3390/ijerph16040602

23. Usman H, Victor V, Waziri I (2018) Qualitative phytochemical screening and in vitro antimicrobial activities of Solanum americanum mill. Arid Zone J Eng Technol 14:104-110

24. Offor SJ, Mbagwu HO, Orisakwe OE (2019) Improvement of lead acetate-induced testicular injury and sperm quality deterioration by Solanum anomalum thonn. ex. schumach fruit extracts in albino rats. J Family Reprod Health 13:98-108

25. Bermejo DV, Ibáñez E, Reglero G, Fornari T (2016) Effect of cosolvents (ethyl lactate, ethyl acetate and ethanol) on the supercritical $\mathrm{CO}_{2}$ extraction of caffeine from green tea. J Supercrit Fluids 107:507-512. https://doi.org/10.1016/j.supflu.2015.07.008

26. Carré P, Citeau M, Dauguet S (2018) Hot ethanol extraction: economic feasibility of a new and green process. OCL 25:D206. https://doi.org/10.1051/ocl/2017061

27. Nutrizio M, Maltar-Strmečki N, Chemat F et al (2021) Highvoltage electrical discharges in green extractions of bioactives from oregano leaves (origanum vulgare 1.) using water and ethanol as green solvents assessed by theoretical and experimental procedures. Food Eng Rev 13:161-174. https://doi.org/10.1007/ s12393-020-09231-2

28. González-Félix MA, Mejía-Manzano LA, Barba-Dávila BA, Serna-Saldívar SO, González-Valdez J (2021) optimized and scalable green extraction of pristimerin, an anticancerigen from mortonia greggii, by ethanol-phosphate aqueous two-phase systems. Ind Eng Chem Res 60:5403-5410

29. Ramabulana T, Mavunda RD, Steenkamp PA, Piater LA, Dubery IA, Madala NE (2015) Secondary metabolite perturbations in 
Phaseolus vulgaris leaves due to gamma radiation. Int J Plant Physiol Biochem 97:287-295. https://doi.org/10.1016/j.plaphy. 2015.10.018

30. Knapsack Core system. Available online http://www.knapsackfa mily.com/knapsack_core/top.php (accessed 20 March 2020)

31. Munari CC, de Oliveira PF, Campos JCL, Martins SPDL, Da Costa JC, Bastos JK, Tavares DC (2014) Antiproliferative activity of Solanum lycocarpum alkaloidic extract and their constituents, solamargine and solasonine, in tumor cell lines. J Nat Med 68:236-241. https://doi.org/10.1007/s11418-013-0757-0

32. Chester K, Paliwal S, Khan W, Ahmad S (2017) UPLC-ESI-MS/ MS and HPTLC method for quantitative estimation of cytotoxic glycosides and aglycone in bioactivity guided fractions of Solanum nigrum L. Front Pharmacol 8:1-15. https://doi.org/10.3389/ fphar.2017.00434

33. Doulabi M, Golmakani MT, Ansari S (2020) Evaluation and optimization of microwave-assisted extraction of bioactive compounds from eggplant peel by-product. J Food Process Preserv 44:1-13. https://doi.org/10.1111/jfpp.14853

34. Veggi PC, Martinez J, Meireles MAA (2012) Fundamentals of microwave extraction. In Microwave-assisted extraction for bioactive compounds Springer, Boston, MA., 2012:15-22. https://doi. org/10.1007/978-1-4614-4830-3_2

35. Zhang HF, Yang XH, Wang Y (2011) Microwave assisted extraction of secondary metabolites from plants: current status and future directions. Trends Food Sci Technol 22:672-688. https:// doi.org/10.1016/j.tifs.2011.07.003

36. Alara OR, Abdurahman NH (2019) Microwave-assisted extraction of phenolics from Hibiscus sabdariffa calyces: kinetic modelling and process intensification. Ind Crop Prod 137:528-535. https:// doi.org/10.1016/j.indcrop.2019.05.053
37. Sajid M, Nazal MK, Rutkowska M, Szczepańska N, Namieśnik J, Płotka-Wasylka J (2019) Solid phase micro-extraction: apparatus, sorbent materials, and application. Crit Rev Anal Chem 49:271-288. https://doi.org/10.1080/10408347.2018.1517035

38. Routray W, Orsat V (2012) Microwave-assisted extraction of flavonoids: a review. Food Bioproc Tech 5:409-424. https://doi.org/ 10.1007/s11947-011-0573-Z

39. Valdés A, Burgos N, Jiménez A, Garrigós MC (2015) Natural pectin polysaccharides as edible coatings. Coatings 5:865-886. https://doi.org/10.3390/coatings5040865

40. Danish M, Jing H, Pin Z, Ziyang L, Pansheng Q (2016) A new drying kinetic model for sewage sludge drying in presence of $\mathrm{CaO}$ and NaClO. Appl Therm Eng 106:141-152. https://doi.org/10. 1016/j.applthermaleng.2016.05.191

41. Jia Z, Yang Y, Yang L, Zhang Y, Sun Z (2018) Hydration products, internal relative humidity and drying shrinkage of alkali activated slag mortar with expansion agents. Constr Build Mater 158:198-207. https://doi.org/10.1016/j.conbuildmat.2017.09.162

42. Gardner MS, Kuklenyik Z, Lehtikoski A, Carter KA, McWilliams LG, Kusovschi J, Bierbaum K, Jones JJ, Rees J, Reis G, Pirkle JL, Barr JR (2019) Development and application of a high throughput one-pot extraction protocol for quantitative LC-MS/MS analysis of phospholipids in serum and lipoprotein fractions in normolipidemic and dyslipidemic subjects. J Chromatogr B 1118:137-147. https://doi.org/10.1016/j.jchromb.2019.04.041

Publisher's note Springer Nature remains neutral with regard to jurisdictional claims in published maps and institutional affiliations. 